\title{
THE ATMOSPHERIC LIMITATION ON THE PRECISION OF GROUND-BASED ASTROMETRY
}

\author{
I.S. GUSEVA \\ Pulkovo Observatory \\ 196140 St Petersburg \\ Russia
}

\begin{abstract}
Anomalous refraction remains to be the most critical problem in the meridian astrometry measuring large angles on the sky. I study slow quasi-periodical variations of refraction caused by the processes in the middle and upper atmosphere, such as gravity waves, etc., which can not be detected and calibrated out by use of any on-ground meteorological measurements. For this study, very old observations at large zenith distances of 80 to 90 degrees made by V. Fuss at Pulkovo Observatory in 18671869 [1] were used. The Deeming's method [2] of spectral analysis of data was applied to examine the characteristic variations of refraction in a wide range of periods. Very powerful quasi-periodical processes with periods of $7-8,11-14,18-22,36-44$ minutes and with amplitudes of 0.3 to 0.5 arcsec in the zenith were found when short sets of observations (1-5 days) were considered. They increase random errors of astrometric observations with meridian circles, transit instruments, astrolabes, etc. The periods of very slow variations - 152, 122, 93, 82.5, 73, 61 and 50 days, - are close to the well known periods discovered in other astronomical phenomena, for instance, in solar activity and in Earth rotation. I note also, that some of the long-period variations of refraction may cause quasi-systematic errors in astrometric measurements and catalogues.
\end{abstract}

\section{References}

1. Fuss, V. (1872) Mem.Acad.Imp.Sci.de St.-Petersbourg, T.XVIII, N3.

2. Deeming, T.J. (1975) Astr. Space Sci., 36. 